\title{
A novel antitumor dithiocarbamate compound inhibits the EGFR/AKT signaling pathway and induces apoptosis in esophageal cancer cells
}

\author{
YUN YANG $^{1,2^{*}}$, ZIYIN TIAN $^{1 *}$, XINGHUA ZHAO $^{1 *}$, YA LI $^{3}$ and SHUYAN DUAN ${ }^{1}$ \\ ${ }^{1}$ Department of Biochemistry and Molecular Biology, School of Basic Medical Sciences; \\ ${ }^{2}$ Xinxiang Key Laboratory of Tumor Vaccine and Immunotherapy, Xinxiang Medical University, \\ Xinxiang, Henan 453000; ${ }^{3}$ Department of Gastroenterology, The First Affiliated Hospital of \\ Zhengzhou University, Zhengzhou, Henan 450000, P.R. China
}

Received September 23, 2019; Accepted April 16, 2020

DOI: $10.3892 / \mathrm{ol} .2020 .11638$

\begin{abstract}
Dithiocarbamate has been reported to possess a potent antitumor efficacy against several types of cancer, such as ovarian cancer, breast cancer and hepatocellular carcinoma; however, only a few studies have investigated its inhibitory effect on esophageal cancer. Dipyridylhydrazone dithiocarbamate (DpdtC) is a novel dithiocarbamate derivative that was recently designed, synthesized and evaluated in our previous study. In the present study, the cell growth inhibition and apoptosis induced by DpdtC were measured using the CCK-8 and Annexin V-FITC/propidium iodide staining assays, respectively. Epidermal growth factor receptor (EGFR) signaling pathway and apoptosis related protein levels were examined by western blotting. In vivo effect of DpdtC was evaluated in nude mice bearing KYSE-450 xenograft tumors. The aims of the present study were to further evaluate the antitumor effects of DpdtC on esophageal cancer cells (KYSE-150 and KYSE-450 cells), and to investigate its potential mechanism of action in vitro and in vivo. It was found that DpdtC significantly inhibited KYSE-150 and KYSE-450 cell proliferation by regulating the EGFR/AKT signaling pathway and inducing apoptosis. In addition, this effect was further identified in vivo; DpdtC inhibited the growth of the KYSE-450 esophageal cancer xenografts by regulating the EGFR/AKT signaling pathway. Furthermore, DpdtC did not affect the body weight
\end{abstract}

Correspondence to: Professor Yun Yang or Professor Shuyan Duan, Department of Biochemistry and Molecular Biology, School of Basic Medical Sciences, Xinxiang Medical University, 601 Jinsui Road, Xinxiang, Henan 453000, P.R. China

E-mail: jamesyangyun1@126.com

E-mail: 191023@xxmu.edu.cn

${ }^{*}$ Contributed equally

Key words: esophageal cancer, dithiocarbamate, epidermal growth factor receptor, AKT, apoptosis in mice. Collectively, the present results suggested that DpdtC may be a promising antitumor drug candidate for the treatment of esophageal cancer.

\section{Introduction}

Esophageal cancer is a malignant disease associated with poor prognosis. Furthermore, esophageal squamous cell carcinoma (ESCC) is a type of esophageal carcinoma that is usually located in the upper or middle third part of the esophagus (1), and had a high-incidence rate between $90-120$ per $10^{5}$ population in the 5-year period (1995-1999) in the Henan region in China (2).

Current therapeutic regimens for patients with esophageal cancer include surgery followed by conventional chemotherapy or radiation; however, the 5-year survival percentage remains very low at $<10 \%(2,3)$. Thus, effective therapeutic agents or regimens are urgently required for improving the survival rate of patients with esophageal cancer.

Metal chelators are a class of potential therapeutics that have potent and selective anti-cancer efficacy (4,5). Most cancer cells have an increasing demand for metal ions, such as iron or copper, to maintain an appropriate proliferation rate; therefore, chelators may be a potential treatment regimen for treating cancer types (6,7). Previous studies have reported that metal chelators, such as di-2-pyridyl ketone-4,4-dimethyl-3-thiosemicarbazone (Dp44mT), possess an inhibitory effect against colon cancer and prostate cancer $(8,9)$.

Dithiocarbamates are sulfur-containing compounds with an excellent chelating property toward metal ions that can regulate crucial molecules involved in reactive oxygen species accumulation, cell cycle arrest, apoptosis or autophagy $(6,10,11)$. However, the molecular targets of numerous dithiocarbamates, such as di-2-pyridylhydrazone dithiocarbamate and pyrrolidine dithiocarbamate remain unknown. Dixon et al (8) revealed that Dp44mT may exert its anti-growth activity by inhibiting the oncogenic ERK1/2 signaling pathway. Moreover, Chen et al (9) reported that the iron chelator desferrioxamine (DFO) can inhibit epithelial-mesenchymal transition (EMT) induced by the transforming growth factor- $\beta$ and by elevating the protein expression of $\mathrm{N}$-myc downstream-regulated gene 1 . 
Our previous study synthesized a class of dithiocarbamate derivative, dipyridylhydrazone dithiocarbamate (DpdtC), and assessed its anti-cancer activity on hepatocellular carcinoma cells (6). It was revealed that DpdtC downregulates erb-b2 receptor tyrosine kinase 2 (ERBB2) expression and disrupts the formation of a heterodimer between ERRB2 and epidermal growth factor receptor (EGFR), which further resulted in the inactivation of ERBB2/ERK $1 / 2$ signaling in ERBB2-overexpressed ovarian cancer cells (12).

The EGFR/AKT signaling pathway has an important role in the growth and proliferation of esophageal cancer cells (13). In the present study, the antitumor effects of DpdtC on esophageal cancer cells were evaluated and its potential mechanism of action was investigated, which may be associated with EGFR/AKT signaling pathway inhibition. The present study aimed to identify the potential of DpdtC as a drug candidate for treatment of EGFR-positive esophageal cancer types, which will aid in the clinical development of esophageal cancer treatment.

\section{Materials and methods}

Cell lines and animals. The human esophageal cancer cell lines KYSE-150 and KYSE-450 were purchased from the American Type Culture Collection. Cell were cultured with RPMI-1640 medium (Gibco; Thermo Fisher Scientific, Inc.) supplemented with $10 \%$ Fetal Bovine Serum (Gibco; Thermo Fisher Scientific, Inc.), 2 mmol/l glutamine, $100 \mathrm{IU} / \mathrm{ml}$ penicillin and $100 \mathrm{mg} / \mathrm{ml}$ streptomycin (Invitrogen; Thermo Fisher Scientific, Inc.) in an incubator at $37^{\circ} \mathrm{C}$ with $5 \% \mathrm{CO}_{2}$ for $48 \mathrm{~h}$. Female BALB/c nude mice (age, 5 weeks; weight, $16-19 \mathrm{~g} ; \mathrm{n}=15$ ) were obtained from the Beijing Vital River Laboratory Animal Technology Co., Ltd. All animals were treated in accordance with guidelines of the Committee on Animals of the Xinxiang Medical University and was approved by Biomedical Ethics Committee of Xinxiang Medical University.

In vitro cytotoxicity assays. Firstly, the effects of different treatment times $(24,48$ or $72 \mathrm{~h})$ on the cytotoxicity of DpdtC (Henan International Joint Lab of Recombinant Protein) in esophageal cancer cells was assessed for determining the appropriate treatment time. Esophageal cancer KYSE-150 and KYSE-450 cell lines were treated with $10 \mu \mathrm{M}$ DpdtC for the aforementioned 3 time points $(24,48$ or $72 \mathrm{~h})$ at $37^{\circ} \mathrm{C}$ with $5 \% \mathrm{CO}_{2}$. Cell viability was then tested using the Cell Counting 8 (CCK-8) Kit (Dojindo Molecular Technologies, Inc.) according to the manufacturer's instructions. Next, cells were treated with DpdtC (Henan International Joint Lab of Recombinant Protein) at a series of concentrations, which was diluted from $50 \mu \mathrm{M}$ in a $2 \mathrm{X}$ dilution manner (50, $25,12.5,6.25,3.125,1.5625$ and $0.78125 \mu \mathrm{M})$ at $37^{\circ} \mathrm{C}$ with $5 \% \mathrm{CO}_{2}$ for $48 \mathrm{~h}$. After 2 days, cell viability was determined using CCK-8 Kit (Dojindo Molecular Technologies, Inc.). The percentage of surviving cells was calculated using the following formula: [(A450 of experiment-A450 of background)/(A450 of untreated control-A450 of background)] $\mathrm{x} 100$. The well treated with only medium without DpdtC was the untreated control. $\mathrm{IC}_{50}$ was calculated using non-linear regression analyses utilizing GraphPad Prism 5 software (GraphPad Software, Inc.).
In vivo therapy study. All animal experimentation followed internationally recognized Animal Research: Reporting of in vivo Experiments guidelines (14). Female BALB/c nude (age, 5 weeks) mice were maintained at $22 \pm 2^{\circ} \mathrm{C}$ and $50-60 \%$ humidity in a $12 \mathrm{~h}$ dark/light cycle, with continuous free access to food and water.

KYSE- 450 cells $\left(5 \times 10^{6}\right.$ per mouse) were inoculated subcutaneously into the right flank of the female BALB/c nude mice. When tumor volumes reached an average of $\sim 150 \mathrm{~mm}^{3}$, the mice were randomly divided into 3 groups $(\mathrm{n}=5$ in each group): i) A PBS-treated group as control; ii) a DpdtC-treated group at a low dose $(1 \mathrm{mg} / \mathrm{kg})$; and iii) a DpdtC-treated group at a high dose $(3 \mathrm{mg} / \mathrm{kg})$. Mice were intraperitoneally injected with $200 \mu 110 \mathrm{mM}$ PBS or DpdtC ( 1 or $3 \mathrm{mg} / \mathrm{kg}$ ) four times (day $0,2,4$ and 6) as indicated in the period of 14 days. The dosages of DpdtC were chosen according to our previous study (12). On day 13 post-first injection, the mice were euthanized using carbon dioxide gas $(20 \% / \mathrm{min}$ gradual displacement) and monitored for $5 \mathrm{~min}$ to confirm cardiac arrest, and the tumors were removed for subsequent western blot examination. The tumors were measured with digital calipers, and tumor volumes were calculated using the formula: Volume $=$ Length $\mathrm{x}(\text { Width })^{2} / 2$. Unspecific toxicity evaluation was determined in tumor-bearing nude mice injected with PBS control or DpdtC by monitoring the body weight of these mice at regular intervals during the whole therapeutic period.

Immunoblotting. Western blot analysis was performed according to previously described procedures (15). The sample was the protein extracted from cell pellets or tumor tissues. Cells were treated with DpdtC $(0,10,20$ or $30 \mu \mathrm{M})$ for $24 \mathrm{~h}$ before being lysed. Tumor tissues were removed on day 13 post the first administration of DpdtC. Then, cells or tumor tissues were lysed in lysis buffer $[50 \mathrm{mmol} / \mathrm{l}$ Tris- $\mathrm{HCl}$ $\mathrm{pH} 7.4,150 \mathrm{mmol} / 1 \mathrm{NaCl}, 0.1 \%$ SDS, $1 \%$ Triton $\mathrm{x}-100$ and $0.5 \%$ deoxycholic acid sodium salt (w/v)] supplemented with $2 \mu \mathrm{l} / \mathrm{ml}$ protease inhibitor cocktail (Sigma-Aldrich; Merck $\mathrm{KGaA}$ ) on ice for $30 \mathrm{~min}$ and centrifuged at $12,000 \mathrm{x} \mathrm{g}, 4^{\circ} \mathrm{C}$ for $20 \mathrm{~min}$ to remove cell debris. Subsequently, the protein $(20 \mu \mathrm{g} / \mathrm{well})$ from the cell lysates were separated using a 10\% SDS-PAGE gel and transferred to PVDF membrane for blocking in 5\% skimmed milk for $1 \mathrm{~h} 30 \mathrm{~min}$ at room temperature. Next, the membrane was immunoblotted with respective antibodies against EGFR (1:1,000; cat. no. 54359; Cell Signaling Technology, Inc.), phosphorylated (p)-EGFR (1:1,000; cat. no. 2234; Cell Signaling Technology, Inc.), AKT (1:1,000; cat. no. 10176-2-AP; ProteinTech Group, Inc.), p-AKT (1:2,000; cat. no. 4060; Cell Signaling Technology, Inc.), Poly (ADP-ribose) polymerase (PARP; 1:500; cat. no. BM5118; Boster Biological Technology), BAX (1:1,000; cat. no. 50599; ProteinTech Group, Inc.), Bcl-2 (1:1,000; cat. no. 4223; Cell Signaling Technology, Inc.), Bcl-2-binding component 3, isoformed 1/2 (BBC3; 1:1,000; cat. no. 55120; ProteinTech Group, Inc.) or $\beta$-actin (1:5,000; cat. no. ab179467; Abcam), and subsequently with horseradish peroxidase-conjugated goat anti-mouse/rabbit secondary antibodies (1:5,000; cat. no. SA00001-1 or SA00001-2; ProteinTech Group, Inc.) at room temperature for $45 \mathrm{~min}$. After washing the membrane with wash buffer (Tris buffered saline with Tween-20, pH 8.0), 
A<smiles>S=C([Hg])NN=C(c1ccccn1)c1ccccn1</smiles>

B

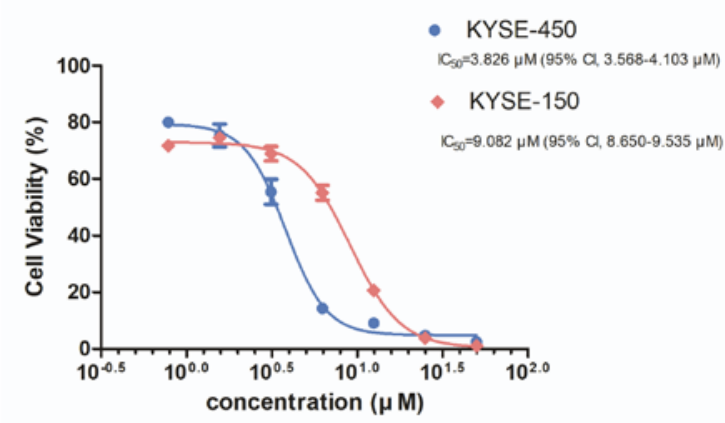

Figure 1. DpdtC markedly reduces the proliferation of esophageal cancer cells in vitro. (A) Chemical structure of DpdtC. (B) Effects of DpdtC on the cytotoxicity of KYSE-450 and KYSE-150 cells. Cells were treated with increasing concentrations of DpdtC $(0.78125,1.5625,3.125,6.25,12.5,25$ and $50 \mu \mathrm{M})$ for $48 \mathrm{~h}$ and then viabilities were determined using Cell Counting Kit- 8 assay. All samples were tested in triplicate. Data are presented as the mean \pm SD. DpdtC, Dipyridylhydrazone dithiocarbamate.

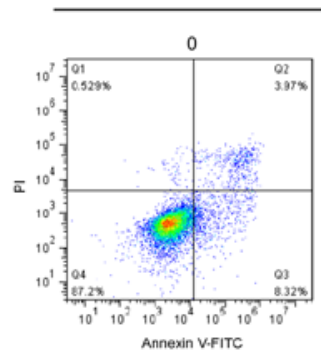

C

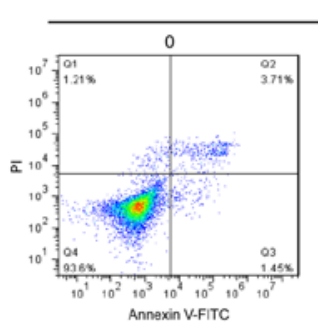

KYSE-450

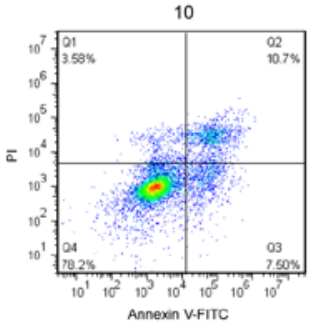

KYSE-150

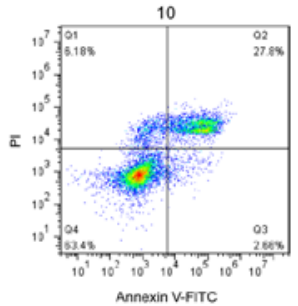

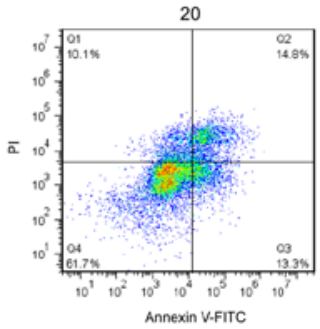

Annexin V-FITC

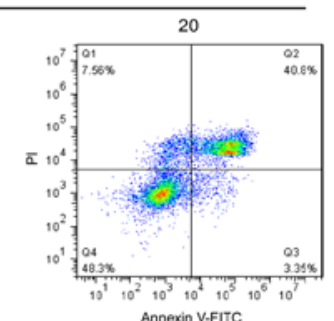

B

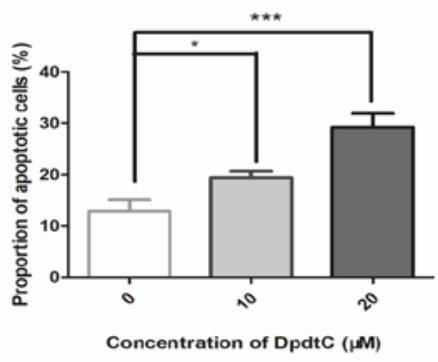

D

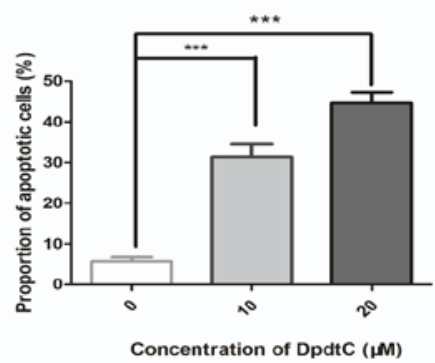

Figure 2. DpdtC induces apoptosis in both KYSE-450 and KYSE-150 cells. The number of apoptotic KYSE-450 cells following treatment with DpdtC (0, 10 or $20 \mu \mathrm{M}$ ) for $20 \mathrm{~h}$ was determined using (A) flow cytometry and the results were analyzed (B) statistically. (C) The number of apoptotic KYSE-150 cells following treatment with DpdtC $(0,10$ or $20 \mu \mathrm{M})$ for $20 \mathrm{~h}$ was determined using flow cytometry and the results were analyzed (D) statistically. Data are presented as the mean $\pm \mathrm{SD}$. ${ }^{*} \mathrm{P}<0.05,{ }^{* * *} \mathrm{P}<0.001$. DpdtC, Dipyridylhydrazone dithiocarbamate; PI, propidium iodide.

the bands were detected using the sensitive ECL reagent (GE Healthcare Life Sciences), and visualized using a ChemiDoc imaging system (Bio-Rad Laboratories, Inc.). Relative densitometry analysis of protein expression level was normalized to control $\beta$-actin antibody using Image $\mathbf{J}$ software v.1.46 (National Institute of Health).

Apoptosis analysis. Apoptosis analysis was performed as previously described (16). For flow cytometry analysis, KYSE-450 or KYSE-150 cells (1x10/well) were plated in 6-well plate and treated with $\operatorname{DpdtC}(0,10$ or $20 \mu \mathrm{M})$ for $20 \mathrm{~h}$ at $37^{\circ} \mathrm{C}$. The cells were then labeled with Annexin V $(20 \mu \mathrm{g} / \mathrm{ml})$ and propidium iodide (PI) $(50 \mu \mathrm{g} / \mathrm{ml})$ (Dojindo Molecular Technologies, Inc.) at room temperature for $15 \mathrm{~min}$. Apoptotic rates were analyzed using a FACSCalibur flow cytometer (BD Biosciences) and calculated using FlowJo software v.7.6.1 (Treestar, Inc.). The rate of early apoptosis was calculated by Annexin V (+) and PI (-), while the rate of late apoptosis was calculated by Annexin V (+) and PI (+).

Statistical analysis. Statistical analysis was performed with GraphPad Prism software v.5.0.1 (GraphPad Software, Inc.). All experiments were repeated 3 times. Numerical values were expressed as the mean \pm standard deviation. For the in vitro and in vivo studies, the differences between the groups were analyzed using one-way ANOVA analysis with a Dunnett's Multiple Comparison post-hoc Test. One-way ANOVA analysis with a Tukey's Multiple Comparison Test was used for analysis of data in Fig. S1. P $<0.05$ was considered to indicate a statistically significant difference. 
A
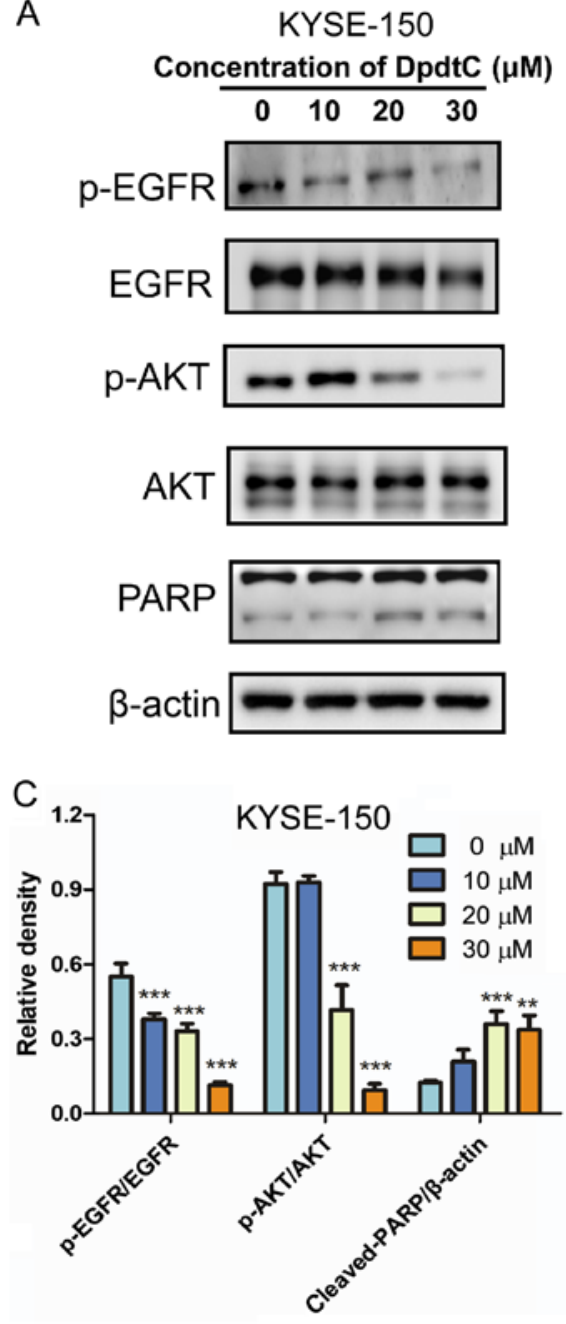

B

KYSE-450

Concentration of DpdtC $(\mu \mathrm{M})$

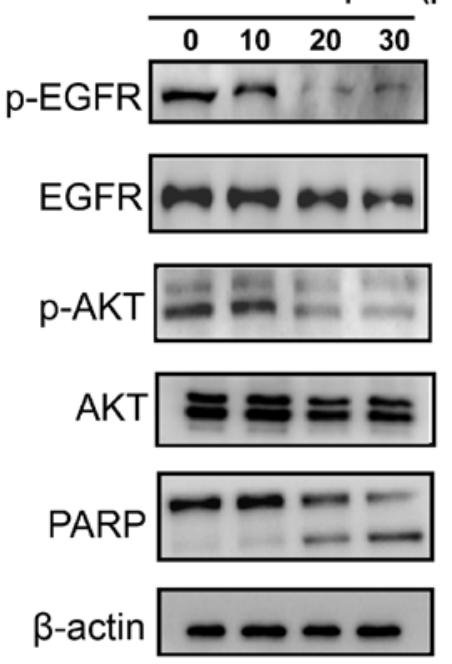

D

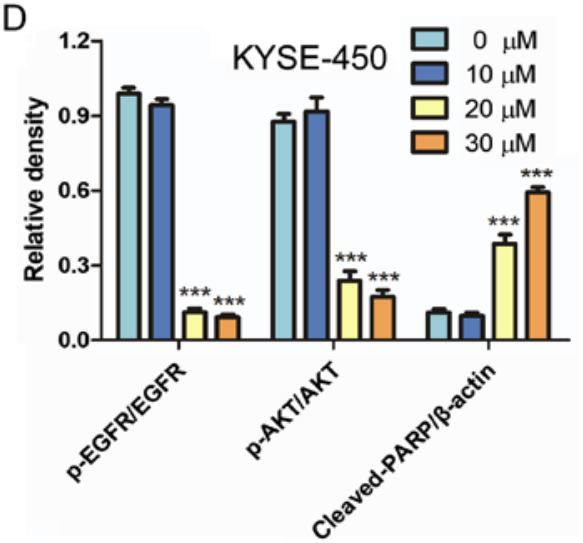

Figure 3. DpdtC inhibits the EGFR/AKT signaling pathway in KYSE-150 and KYSE-450 cells. (A) KYSE-150 and (B) KYSE-450 cells were treated with $\operatorname{DpdtC}(0,10,20$ or $30 \mu \mathrm{M})$ for $24 \mathrm{~h}$ before being evaluated. EGFR, p-EGFR, AKT, p-AKT and PARP were analyzed by immunoblotting assay. Quantification of protein signal intensity in (C) KYSE-150 and (D) KYSE-450 cells and expressed relative to the $\beta$-actin or total EGFR/AKT protein using ImageJ software. Data are presented as the mean $\pm \mathrm{SD}$ of three independent experiments. ${ }^{* *} \mathrm{P}<0.01,{ }^{* * *} \mathrm{P}<0.001$ vs. untreated control cells. DpdtC, Dipyridylhydrazone dithiocarbamate; p-, phosphorylated; PARP, Poly (ADP-ribose) polymerase; EGFR, epidermal growth factor receptor.

\section{Results}

DpdtC exhibits growth inhibitory effects against esophageal cancer cells in vitro. The cytotoxicity of DpdtC against EGFR-overexpressed KYSE-150 and KYSE-450 cells was examined $(2,13)$. The chemical structure of DpdtC is presented in Fig. 1A. Based on the preliminary experiments, $48 \mathrm{~h}$ treatment time with DpdtC was selected for the following CCK-8 assay (Fig. S1). It was demonstrated that DpdtC had a concentration-dependent inhibitory effect in both KYSE-150 and KYSE- 450 cells treatment for $48 \mathrm{~h}$. $\mathrm{IC}_{50}$ for KYSE-450 and KYSE-150 cells was $3.826 \mu \mathrm{M}(95 \% \mathrm{CI}, 3.568-4.103 \mu \mathrm{M})$ and $9.082 \mu \mathrm{M}(95 \% \mathrm{CI}, 8.650-9.535 \mu \mathrm{M})$, respectively (Fig. 1B).

DpdtC induces apoptosis in both KYSE-150 and KYSE-450 cells. Subsequently, whether DpdtC induces apoptosis was investigated in KYSE-150 and KYSE-450 cells. The percentage of apoptotic cells was determined using flow cytometry following Annexin V and PI staining. The results suggested that the apoptotic percentage was significantly increased in both KYSE-150 and KYSE-450 cells treated with an increasing concentration of DpdtC (Fig. 2). Moreover, a significant cleavage of PARP, a classical apoptotic initiator, was observed in both cancer cells treated with DpdtC (Fig. 3). Interestingly, KYSE-450 cells appeared to be more sensitive to DpdtC treatment in the cytotoxicity assay, while the apoptosis percentage of KYSE-450 cells was lower compared with that in the KYSE-150 cells at the same concentration of DpdtC (Figs. 1 and 2).

DpdtC inhibits the EGFR/AKT signaling pathway in KYSE-150 and KYSE-450 cells. The expression of EGFR is increased in esophageal cancer cells KYSE-150 and KYSE-450 $(2,13)$. In the present study, a significant decrease in the phosphorylation levels of EGFR and AKT was identified in both KYSE-150 and KYSE-450 cells treated with DpdtC. Moreover, this decrease was dose-dependent (Fig. 3).

DpdtC inhibits the growth of KYSE-450 tumor xenografts. To evaluate the inhibitory activity of DpdtC in vivo, the therapeutic effect of DpdtC was examined in nude mice with KYSE-450 xenograft tumors. It was found that DpdtC significantly 
A

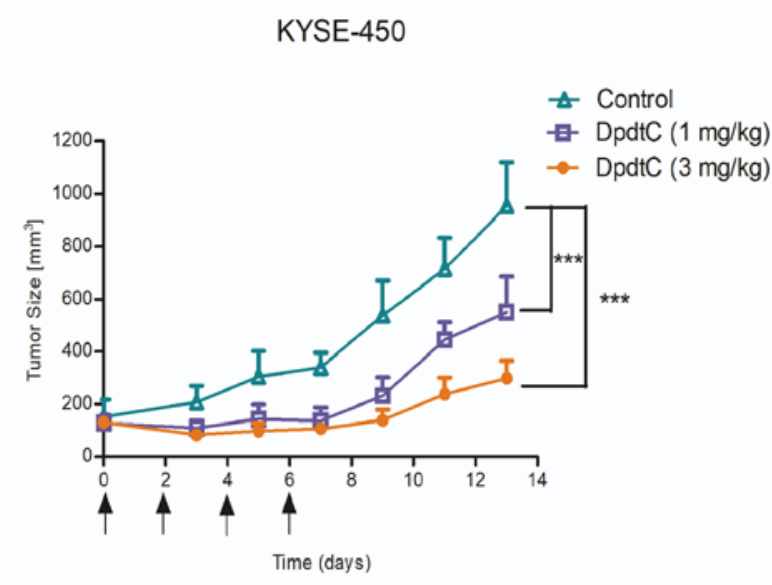

B

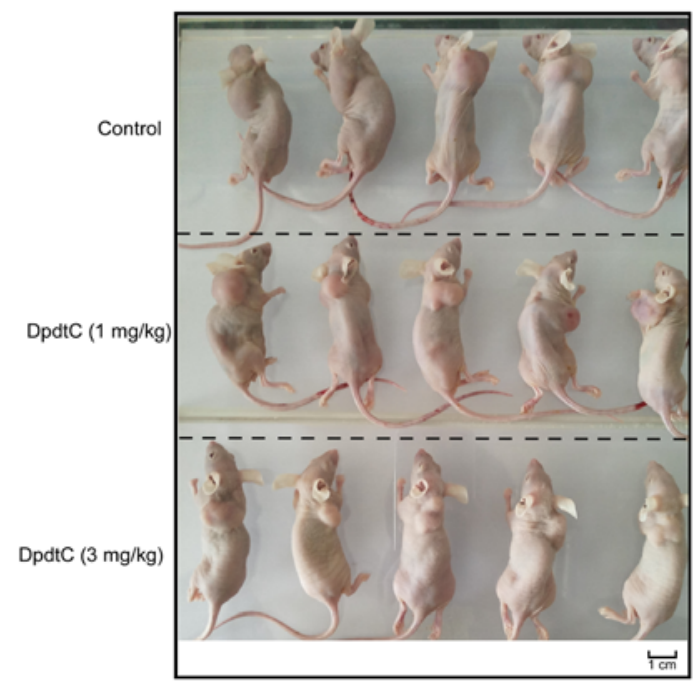

C

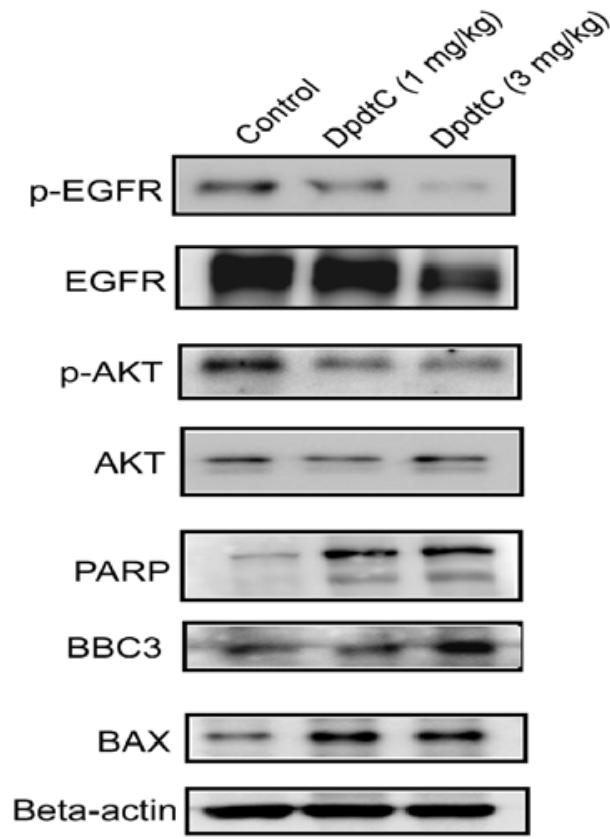

D

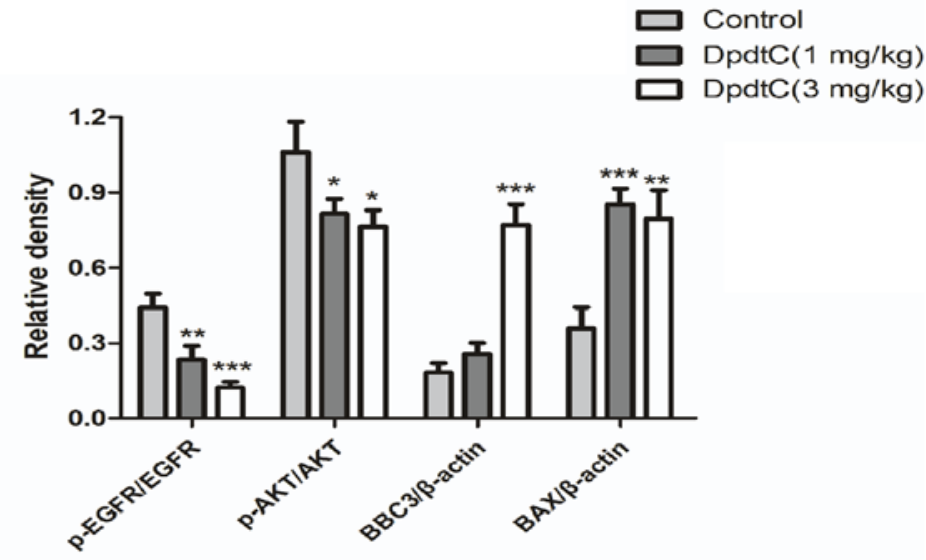

Figure 4. In vivo effects of DpdtC in the KYSE-450 tumor-bearing nude mice. (A) Mean tumor volumes of mice xenografted with KYSE-450 cells and treated with different doses of DpdtC ( 1 or $3 \mathrm{mg} / \mathrm{kg}$ ). $\mathrm{n}=5$. The black arrows indicate the DpdtC treatment times. (B) KYSE- 450 tumor-bearing mice were imaged and tumors were removed on day 13 post-administration. Tumor tissues were isolated from KYSE-450 xenografts following treatment with control (PBS) or DpdtC (1 or $3 \mathrm{mg} / \mathrm{kg})$, then the expression levels of EGFR, p-EGFR, AKT, p-AKT, PARP, BBC3, BAX and $\beta$-actin were determined using (C) western blot analysis and the results were (D) quantified and the protein levels expressed relative to the $\beta$-actin or total EGFR/AKT protein using ImageJ software. Data are presented as the mean $\pm \mathrm{SD}$ of three independent experiments. ${ }^{*} \mathrm{P}<0.05,{ }^{* *} \mathrm{P}<0.01,{ }^{* * * *} \mathrm{P}<0.001$ vs. control group. DpdtC, Dipyridylhydrazone dithiocarbamate; p-, phosphorylated; PARP, Poly (ADP-ribose) polymerase; EGFR, epidermal growth factor receptor; BBC3, Bcl-2-binding component 3.

decreased tumor growth compared with that in the control-PBS treatment group (Fig. 4A and B). Moreover, while both concentrations of DpdtC reduced the growth of tumors, the reduction was higher with $3 \mathrm{mg} / \mathrm{kg}$ DpdtC. In addition, DpdtC treatment did not result in loss in body weight (Fig. S2). Collectively, the results suggested that DpdtC had a dose-dependent inhibitory effect against KYSE-450 esophageal cancer in vivo.

DpdtC induces apoptosis and inhibits the EGFR/AKT signaling pathway in vivo. To further assess the mechanism of action of DpdtC in vivo, tumor samples from treated mice were collected and analyzed using an immunoblotting assay. Related apoptotic markers, including PARP, BBC3 and BAX were also examined to evaluate the apoptosis level in vivo. It was found that the expression levels of p-EGFR and p-AKT in tumors were significantly decreased compared with that in the control-PBS treatment group. Furthermore, PARP was significantly cleaved, while $\mathrm{BBC} 3$ and BAX expression levels were significantly elevated in the DpdtC-treated groups (Fig. 4C and D).

\section{Discussion}

Metal chelators possess a potent and targeted antitumor activity against a variety of types of cancer including myeloid 
leukemia, hepatocellular carcinoma and breast cancers etc. $(6,17-19)$. Previous studies have reported that Dp44mT or DFO may inhibit cancer cell proliferation by regressing multiple signaling pathways including transforming growth factor- $\beta$, AKT, ERK and c-Abelson murine leukemia viral oncogene homolog 1-adaptor molecule CrkII pathways related to tumor progression and metastasis $(4,20,21)$. Dithiocarbamates are sulfur-containing compounds with a strong chelating ability toward metal ions $(11,22)$. Moreover, their derivatives, such as the synthetic gold(III) dithiocarbamate and pyrrolidine derivative of dithiocarbamate may be inhibitors targeting NF- $\kappa \mathrm{B}(23)$, inhibitors against proteasome (24), DNA intercalators (25) and inactivators of metal-containing enzymes (26); however, the underlying mechanism remains to be elucidated.

Our previous study characterized and assessed the chemical properties of DpdtC (6). To the best of our knowledge, the present study was the first to examine the antitumor efficacy of DpdtC against esophageal cancer cells and to investigate its mechanism of action. The present results suggested that DpdtC exhibited effective antitumor effects by inhibiting the EGFR/AKT signaling pathway and inducing apoptosis of esophageal cancer cells in vitro and in vivo. Furthermore, it was found that DpdtC induced the downregulation of EGFR at a relatively high concentration $(30 \mu \mathrm{M})$ in vitro or at a high dose $(3 \mathrm{mg} / \mathrm{kg})$ in vivo, which suggested that the growth inhibition caused by DpdtC may be associated with EGFR downregulation. In addition, the results suggested that treatment with DpdtC was well tolerated by the mice, without affecting their body weights. Therefore, it was hypothesized that DpdtC exerted its antitumor effects primarily by suppressing the EGFR/AKT signaling pathway. DpdtC has a relative weaker potency on inducing apoptosis in KYSE-450 cells compared with that in the KYSE-150 cells; however, it may more effectively inhibit the phosphorylation of EGFR and AKT in KYSE-450 cells compared with that in KYSE-150 cells. Collectively, the results suggested that DpdtC may exert its effects by inhibiting the EGFR/AKT signaling pathway and inducing apoptosis, thus suggesting the potential of DpdtC as a drug candidate for the treatment of EGFR-positive esophageal cancer types.

Currently, esophageal cancer lacks potent treatment regimens, and the 5-year survival rate is $<10 \%$, thus the development of more effective therapeutic agents is required (3). Our previous study developed a novel EGFR-targeted antibody-drug, denoted as PT that exerts antitumor effects on esophageal cancer types by inhibiting the EGFR/ERK1/2 pathway and inducing apoptosis via blockade of the nuclear factor erythroid 2-related factor 2/Kelch-like ECH-associated protein 1 pathway (13). The present study evaluated in vivo the novel drug, DpdtC which has the potential to be used in clinical treatment. Moreover, we hypothesized that the combination of EGFR-targeted antibody drugs with DpdtC could achieve greater antitumor effects by synergistically inhibiting the EGFR downstream signaling pathway and inducing apoptosis in esophageal cancer types, which will be further investigated in future studies.

In conclusion, the present study identified a promising anti-cancer agent, DpdtC, which targets the EGFR/AKT pathway and induces apoptosis, and thus has potential to be a novel drug candidate in treating esophageal cancer types.

\section{Acknowledgements}

The authors would like to thank Professor Changzheng Li (School of Basic Medical Sciences, Xinxiang Medical University) for his technical assistance on the design of the study.

\section{Funding}

This study was supported by grants from the Natural Science Foundation of China(grant nos. 81703054 and 81803076), Henan Provincial Medical Science and Technology Research Project (grant no. SB201901064), Science and Technology Project for Young Talents of Henan Province (grant no. 2020HYTP048), Key Project of School of Basic Medical Sciences in Xinxiang Medical University (grant nos. JCYXYKY201901 and JCYXYKY201907), Key Science and Technology Program of Henan Province (grant nos. 192102310414, 182102310259 and 182102310436) and Innovation Project of Graduate in Xinxiang Medical University (grant no. YJSCX201933Y).

\section{Availability of data and materials}

The datasets used and/or analyzed during the current study are available from the corresponding author on reasonable request.

\section{Authors' contributions}

YY and SD designed the study. YY, ZT, XZ and YL collected the data and performed the experiments. YY and SD performed the statistical analyses. YY and SD wrote and revised the manuscript. All authors read and approved the final manuscript.

\section{Ethics approval and consent to participate}

All animal experiments were approved by the Biomedical Ethics Committee of Xinxiang Medical University.

\section{Patient consent for publication}

Not applicable.

\section{Competing interests}

The authors declare that they have no competing interests.

\section{References}

1. Wu C, Li D, Jia W, Hu Z, Zhou Y, Yu D, Tong T, Wang M, Lin D, Qiao Y, et al: Genome-wide association study identifies common variants in SLC39A6 associated with length of survival in esophageal squamous-cell carcinoma. Nat Genet 45: 632-638, 2013.

2. Guo XF, Zhu XF, Yang WC, Zhang SH and Zhen YS: An EGFR/HER2-Bispecific and enediyne-energized fusion protein shows high efficacy against esophageal cancer. PLoS One 9: e92986, 2014

3. Campbell NP and Villaflor VM: Neoadjuvant treatment of esophageal cancer. World J Gastroenterol 16: 3793-3803, 2010. 
4. Kovacevic Z, Chikhani S, Lovejoy DB and Richardson DR: Novel thiosemicarbazone iron chelators induce up-regulation and phosphorylation of the metastasis suppressor N-myc down-stream regulated gene 1: A new strategy for the treatment of pancreatic cancer. Mol Pharmacol 80: 598-609, 2011.

5. Wang J, Yin D, Xie C, Zheng T, Liang Y, Hong X, Lu Z, Song X, Song R, Yang H, et al: The iron chelator Dp44mT inhibits hepatocellular carcinoma metastasis via N-Myc downstream-regulated gene 2 (NDRG2)/gp130/STAT3 pathway. Oncotarget 5: 8478-8491, 2014.

6. Wang T, Fu Y, Huang T, Liu Y, Wu M, Yuan Y, Li S and Li C: Copper ion attenuated the antiproliferative activity of Di-2-pyridylhydrazone dithiocarbamate derivative; however, there was a lack of correlation between ros generation and antiproliferative activity. Molecules 21: pii: E1088, 2016.

7. Fu Y, Liu Y, Wang J, Li C, Zhou S, Yang Y, Zhou P, Lu C and Li C: Calcium release induced by 2-pyridinecarboxaldehyde thiosemicarbazone and its copper complex contributes to tumor cell death. Oncol Rep 37: 1662-1670, 2017.

8. Dixon KM, Lui GY, Kovacevic Z, Zhang D, Yao M, Chen Z, Dong Q, Assinder SJ and Richardson DR: Dp44mT targets the AKT, TGF- $\beta$ and ERK pathways via the metastasis suppressor NDRG1 in normal prostate epithelial cells and prostate cancer cells. Br J Cancer 108: 409-419, 2013.

9. Chen Z, Zhang D, Yue F, Zheng M, Kovacevic Z and Richardson DR: The iron chelators Dp44mT and DFO inhibit TGF- $\beta$-induced epithelial-mesenchymal transition via up-regulation of N-Myc downstream-regulated gene 1 (NDRG1). J Biol Chem 287: 17016-17028, 2012.

10. Orrenius S, Nobel CS, van den Dobbelsteen DJ, Burkitt MJ and Slater AF: Dithiocarbamates and the redox regulation of cell death. Biochem Soc Trans 24: 1032-1038, 1996.

11. Buac D, Schmitt S, Ventro G, Kona FR and Dou QP: Dithiocarbamate-based coordination compounds as poten proteasome inhibitors in human cancer cells. Mini Rev Med Chem 12: 1193-1201, 2012.

12. Yang Y, Liu Y, Guo R, Fu Y, Zhang Z, Zhang P, Zhou P, Wang T, Huang T, Li X and Li C: The novel dithiocarbamate, DpdtC suppresses HER2-overexpressed cancer cells by up-regulating NDRG1 via inactivation of HER2-ERK $1 / 2$ signaling. Sci Rep 8 : 3398, 2018

13. Yang Y, Tian Z, Ding Y, Li X, Zhang Z, Yang L, Zhao F, Ren F and Guo R: EGFR-targeted immunotoxin exerts antitumor effects on esophageal cancers by increasing ROS accumulation and inducing apoptosis via inhibition of the Nrf2-keap1 pathway. J Immunol Res 2018: 1090287, 2018.

14. Jones-Bolin S: Guidelines for the care and use of laboratory animals in biomedical research. Curr Protoc Pharmacol Appendix 4: Appendix 4B, 2012.

15. Yang Y, Guo R, Tian X, Zhang Z, Zhang P, Li C and Feng Z: Synergistic anti-tumor activity of Nimotuzumab in combination with Trastuzumab in HER2-positive breast cancer. Biochem Biophys Res Commun 489: 523-527, 2017.
16. Zhang L, Jiang G, Yao F, He Y, Liang G, Zhang Y, Hu B, Wu Y, $\mathrm{Li} \mathrm{Y}$ and Liu H: Growth inhibition and apoptosis induced by osthole, a natural coumarin, in hepatocellular carcinoma. PLoS One 7: e37865, 2012.

17. Yuan J, Lovejoy DB and Richardson DR: Novel di-2-pyridylderived iron chelators with marked and selective antitumor activity: In vitro and in vivo assessment. Blood 104: 1450-1458, 2004.

18. Whitnall M, Howard J, Ponka P and Richardson DR: A class of iron chelators with a wide spectrum of potent antitumor activity that overcomes resistance to chemotherapeutics. Proc Natl Acad Sci USA 103: 14901-14906, 2006.

19. Ohyashiki JH, Kobayashi C, Hamamura R, Okabe S, Tauchi T and Ohyashiki K: The oral iron chelator deferasirox represses signaling through the mTOR in myeloid leukemia cells by enhancing expression of REDD1. Cancer Sci 100: 970-977, 2009.

20. Le NT and Richardson DR: Iron chelators with high antiproliferative activity up-regulate the expression of a growth inhibitory and metastasis suppressor gene: A link between iron metabolism and proliferation. Blood 104: 2967-2975, 2004.

21. Liu W, Yue F, Zheng M, Merlot A, Bae DH, Huang M, Lane D, Jansson P, Lui GY, Richardson V, et al: The proto-oncogene $\mathrm{c}$-Src and its downstream signaling pathways are inhibited by the metastasis suppressor, NDRG1. Oncotarget 6: 8851-8874, 2015.

22. Li C, Liu Y, Fu Y, Huang T and Kang L: The antiproliferative activity of di-2-pyridylketone dithiocarbamate is partly attributed to catalase inhibition: Detailing the interaction by spectroscopic methods. Mol Biosyst 13: 1817-1826, 2017.

23. Schreck R, Meier B, Mannel DN, Droge W and Baeuerle PA Dithiocarbamates as potent inhibitors of nuclear factor kappa $B$ activation in intact cells. J Exp Med 175: 1181-1194, 1992.

24. Milacic V, Chen D, Ronconi L, Landis-Piwowar KR, Fregona D and Dou QP: A novel anticancer gold(III) dithiocarbamate compound inhibits the activity of a purified $20 \mathrm{~S}$ proteasome and $26 \mathrm{~S}$ proteasome in human breast cancer cell cultures and xenografts. Cancer Res 66: 10478-10486, 2006.

25. Ronconi L, Marzano C, Zanello $P$, Corsini M, Miolo G, Maccà C, Trevisan A and Fregona D: Gold(III) dithiocarbamate derivatives for the treatment of cancer: Solution chemistry, DNA binding, and hemolytic properties. J Med Chem 49: 1648-1657, 2006.

26. Nobel CS, Burgess DH, Zhivotovsky B, Burkitt MJ, Orrenius S and Slater AF: Mechanism of dithiocarbamate inhibition of apoptosis: Thiol oxidation by dithiocarbamate disulfides directly inhibits processing of the caspase- 3 proenzyme. Chem Res Toxicol 10: 636-643, 1997.

This work is licensed under a Creative Commons Attribution-NonCommercial-NoDerivatives 4.0 International (CC BY-NC-ND 4.0) License. 